\title{
Where less is more; a case based discussion on the damage control resuscitation, a fundamental concept in the current management of major trauma
}

\author{
Seneviratne RW, Kumara MMAJ \\ Department of Surgery, Faculty of Medicine, University of Ruhuna, Galle, Sri Lanka.
}

Correspondence: Dr. Ranjana Seneviratne

e-mail: ranjanamst@yahoo.com

\section{Introduction}

Systemic inflammatory response syndrome (SIRS) is a condition recognized recently. Inadequacies in resuscitation limited the survival of patient in the initial hours after major trauma until a relatively recent development changed the picture. Coagulopathy, acidosis and hypothermia (collectively called fatal triad) leading to SIRS followed by organ failure and even death is one of the most feared sequels of major trauma. Damage Control Resuscitation (DCR) was developed In order to prevent this 'dark' sequence by addressing its initiation during the immediate post-major trauma period. We discuss this novel and lifesaving concept using our experience in the successful management of a young man who sustained a major trauma.

Components of DCR are permissive hypotension until haemorrhage control, haemostatic resuscitation, limited use of crystalloids to avoid dilutional coagulopathy and damage control (Salvage) surgery to preserve physiology as well as to control haemorrhage.

DCR is the principle concept employed in the resuscitation of major trauma. This procedure saves lives increasingly and has gained acceptance globally. Our patient after suffering from a major trauma was successfully managed in a reasonably developed still less than ideal setting. Although perfection should be the aim, emergency health care systems in developing countries like Sri Lanka must embrace this concept and practice it as much as feasible for the benefit of many injured.

\section{Case report}

A 28 year-old three wheeler driver was admitted to Emergency Trauma Centre, Teaching Hospital, Karapitiya, Galle, about 30 minutes after his vehicle met with a head-on collision with a bus. Handle of the wheeler turned right angle during accident with its end creating an imprint on mid upper abdomen.

Initial inquiry did not reveal any airway or breathing problems but patient was in haemorrhagic shock with pulse rate of 125 beats per minute, blood pressure of $85 / 60 \mathrm{mmHg}$ and distended abdomen. Initial GCS was 14. His spine was immobilized although he did not have any evidence of spinal injury. He had no significant limb or other injuries except a few scattered abrasions.

Patient was managed at the resuscitation bay. Extended focused abdominal sonography (eFAST) revealed the presence of intraperitoneal free fluid. Patient was given $1 \mathrm{~L}$ of normal saline followed by one unit each of whole blood, fresh frozen plasma (FFP) and platelets. Systolic blood pressure was maintained around $100 \mathrm{mmHg}$. He was taken to emergency theatre about 60 minutes after the injury for an exploratory laparotomy. Approximately $1.5 \mathrm{~L}$ of intraperitoneal blood was removed. The patient had $5 \mathrm{~cm}$ transverse laceration across stomach cutting across gastroepiploic vessels and lowers half of the body of pancreas sparing main pancreatic duct. He also had severe bruising of $3^{\text {rd }}$ part of duodenum and mesentery of transverse colon. In addition large left sided retroperitoneal haematoma was found. Spleen was intact but the patient had $3 \mathrm{~cm}$ through and through laceration involving left lobe of the liver. After initial packing, the gastric laceration was 
repaired in two layers with $3 / 0$ polygalactin. Pancreatic tear was over sawn with the same material. Gastroepiploic vessels and some minor bleeders were tied off. Exploration of haematoma around the 3rd part of the duodenum did not reveal any perforations. Left sided retroperitoneal haematoma was not explored as per trauma guidelines. Patient required 1 unit each of blood, FFP and platelets during the surgery and had $1 \mathrm{~L}$ of Hartmann solution afterwards. Tranexamic acid was administered. Systolic blood pressure was kept between 100 and 110 during surgery and for 24 hours afterwards. Abdomen was closed with two drainsone each for lesser sac and pelvis.

Postoperatively patient was admitted to the intensive care unit where he stayed for seven days followed by another 12 days stay in high dependency unit. Patient was treated with nasogastric drainage and octreotide post operatively and serum amylase was repeatedly checked. He did not develop pancreatitis. Haemoglobin and other parameters did not drop after patient had one unit each of blood, FFP and Platelets on day two postoperative. Due to repaired large gastric laceration and concern about severely bruised duodenal wall succumbing to a delayed perforation, patient was maintained on parenteral feeding and nothing by mouth regime until $10^{\text {th }}$ postoperative day. Gastrografin study via NG tube confirmed the patency of the duodenum after which fluid and solids were gently reintroduced. Pelvic drain was removed on the $5^{\text {th }}$ postoperative day but drain in the lesser sac was removed only 13 days after oral feeding was established. CT - Intravenous urogram was performed on the $15^{\text {th }}$ postoperative day due to initial suspicion of damage to the left kidney. It showed a non functioning necrotic fragmented left kidney indicating total avulsion at the time of injury. As his general condition improved steadily he was mobilized slowly and discharged home after a total of 28 days in the hospital.

\section{Discussion}

This young man presented in Class III haemorrhagic shock, intraperitoneal contamination and multiple intra abdominal organ injury posed a major challenge to clinicians. Permissive hypotension with limited crystalloids and early use of blood and blood components all warmed to body temperature as well as administration of tranexamic acid probably minimized the derangement of coagulation system and bleeding. The maintenance of relative hypotension during surgery and first 24 hours postoperatively too contributed to this favorable outcome. Other important management concepts employed include non-exploration of lateral retroperitoneal haematoma as well as a cautious approach to enteral feeding in the face of potential gastric anastomotic leak and the duodenal blowout. Octreotide and delayed feeding was also intended to minimize the risk of acute pancreatitis.

The concept of damage control in acute management of trauma originated from the realization that improper resuscitation along with prolonged and complex surgical procedures in a patient with major injuries worsens ones chances. It was found that such patients do better when initial surgery was confined to life saving temporalizing measures allowing physiology to stabilize the patient in an intensive care unit for 24-48 hours before definitive surgery. This earned the name Damage control surgery (DCS) or salvage surgery. Damage control, a naval term, is defined as "the capacity of a ship to absorb damage and maintain mission integrity" by permitting only minimal emergency repairs at middle of the sea enabling the ship to reach the harbor docks for definitive repairs (1).

Physiological insult by single overwhelming hit or two successive hits leads to the development of fatal triad which consists of Coagulopathy, Hypothermia and Acidosis. This in turn pushes the patient towards Systemic Inflammatory Response Syndrome (SIRS), multi-organ failure (MOF) and even Death. This dreaded path should be avoided in the management of trauma. The first hit corresponds to the magnitude of the initial injury while the second hit can be a massive blood transfusion, prolonged hypoxia, severe hypotension, lengthy surgery etc. Reversal of the effects of the first hit and prevention of a second hit are the conceptual background to DCR. Orderly rapid resuscitation guided by Advanced Trauma and Life support (ATLS) protocol plays a valuable role in this context.

It is said that medical science is the only field benefited from warfare. The concept of DCR which has emerged over the last 10 years is an offshoot of recent war experiences in middle east by armed forces of US and UK (2). This evolved naturally from the concept of DCS which has been in existence for 
over the last 20 years. Now DCS is considered a component of DCR. DCR can be defined as a systematic approach to patients with exsanguinating trauma, incorporating several strategies to reduce morbidity and mortality. This objective is achieved via minimizing the occurrence of fatal triad of hypothermia, acidosis and coagulopathy.

Hypothermia has multi factorial origin in a trauma patient. It can be due to exposure to cold at scene, during transport or in the operating theatre. Administration of cold fluids and blood products as well as effects of drugs for sedation and anesthesia too contributes to this. Hypothermia increases bleeding by impeding platelet adhesion, dysregulation of coagulation factors and by interfering with fibrinolysis. Countermeasures include external rewarming such as use of blankets as well as internal rewarming strategies including warming of IV fluids.

Acidosis is again has multi factorial origin. Hypoxia, inadequate tissue perfusion, lactic acidosis as well as the use of excessive normal saline resuscitation with its supra physiological chloride levels all contribute. Acidosis decreases coagulation factor activity. Clinically the degree of base deficit and lactate level on admission tend to strongly correlate with worsened patient mortality. Correction of hypoxia and restoration of circulation are the best countermeasure against acidosis.

Coagulopathy in a trauma patient which is termed trauma induced coagulopathy (TIC) by Brohi et al (2003) is caused by multiple independent but interacting mechanisms(3). Acute Traumatic coagulopathy (ATC), the endogenous component of TIC is initiated within 4-5 minutes of injury and has an association with the magnitude of trauma and shock. This is characterized by isolated factor $\mathrm{V}$ inhibition, impaired platelet function, dysfibrinogenemia, hyperfibrinolysis and systemic anticoagulation $(4,5)$. Exogenous causes of TIC include acidosis, hypothermia, and dilution by hypocoagulable fluid etc. INR of 1.3 and above is an indicator of coagulopathy (6). As coagulopathy appears to be the main obstacle of a patient trying to recover from the effects of major trauma, much research has been directed towards rapid diagnosis and early direct interventions.

\section{Components of DCR are,}

A) Permissive hypotension until haemorrhage control

B) Haemostatic resuscitation

C) Limited use of crystalloids and colloids to avoid dilutional coagulopathy.

D) Damage control (Salvage) surgery to preserve physiology and to control haemorrhage

\section{(A) Permissive hypotension until haemorrhage control}

Achieving normotension with aggressive fluid resuscitation can be harmful in trauma patients without haemorrhage control as it can increase haemorrhage via clot dislodgement and coagulopathy. Minimal normotension or permissive hypotension where systolic blood pressure is maintained around $90 \mathrm{mmHg}$ by controlled administration of fluid and blood components reduces bleeding and is preferred until haemorrhage is controlled. Head injury is a possible exception where systolic blood pressure around $100 \mathrm{mmHg}$ is accepted.

In our patient we maintained hypotension until about 24 hours after surgery giving opportunity for significant intraabdominal raw surfaces and retroperitoneal haematomas to stop oozing. In our opinion more studies are in order to search the value of permissive hypotension extending beyond surgery.

\section{(B) Haemostatic resuscitation}

Those who will require massive blood transfusion $(>10$ units within 24 hours) should be anticipated with the aid of scoring systems such as $A B C$ (Assessment of blood consumption) and timely activation of Massive Blood transfusion Protocol (MTP) when signaled. Packed red cells, Fresh frozen plasma, platelets and calcium are main the components of MTP. Some of the adjuncts include Recombinant Factor VIIa, cryoprecipitate and tranesmic acid. Our patient probably had benefit of the latter which is proven to be useful by many studies (13). In ideal circumstances the use of type and quantity of blood and blood components during resuscitation, surgery and early postoperative period should be guided by use of thrombo-elastometry 
providing objective timely information (12). In the absence of this facility, the use of packed red cells, fresh frozen plasma and platelets in ratio of $1: 1: 1$ is shown by studies as the second best alternative (13). Our use of this protocol albeit using whole blood instead of packed cells did minimize the transfusion requirements and aided the survival of our patient. MTP is recognized to be economical on blood transfusion services. This was favored in our case as the patient who had lost 1.5 to $2 \mathrm{~L}$ of blood (Class III shock) only required three units each of whole blood, FFP and platelets.

\section{(C) Limited Use of crystalloids and colloids to avoid dilutional coagulopathy}

Early activation of MTP avoids excess crystalloid administration (2). This minimizes several associated side effects, including reperfusion injury, increased leukocyte adhesion and inflammation, associated acidosis and resulting acute respiratory distress syndrome, systemic inflammatory response syndrome and multi-organ failure (8-10). Our patient had only $1 \mathrm{~L}$ of normal saline and $1 \mathrm{~L}$ of Hartmann solution during the first 24 hours of resuscitation. It also reduces the incidence of both visceral and abdominal wall edema allowing tension free abdominal wall closure i.e. reducing the incidence of abdominal compartmental syndrome.

\section{(D) Damage control (Salvage) surgery to preserve physiology and control haemorrhage}

Damage control surgery (DCS) aimed to do minimum, least time consuming maneuvers to buy time to restore physiology than the correction of disrupted anatomy. This has led to saving of many lives during the recent wars. Only $10 \%$ of US soldiers wounded during 2003 - 2009 period died compared to $24 \%$ in the Vietnam War. However not all patients with trauma need damage control surgery as first surgery may accomplish goals in a short time. In our patient this was the case. Widely accepted damage control surgery trigger protocols include core temperature less than $35^{\circ} \mathrm{C}, \mathrm{pH}$ less than 7.2 , base deficit greater than 15 and significant coagulopathy (9-12). Some protocols have other conditions such as operating time exceeding 60 minutes.
Tools available for surgeons in DCS cover abdominal trauma, thoracic trauma, fractures, vascular injuries and scope is expanding. The range of maneuvers include packing of bleeding liver and pelvis, rapid stapling of segments of lacerated bowel to prevent contamination, pulmonary tractotomy to access depth and deal with bleeders, external fixation of fractures, use of temporary intravascular shunts, angioembolization and balloon catheters to occlude bleeding vessels.

\section{References}

1. Warfare Manual. Department of the Navy. The Department; Washington(DC): 1996.

2. Rhee P, Koustova E, Alam HB. Searching for the optimal resuscitation method: recommendations for the initial fluid resuscitation of combat casualties. The Journal Trauma 2003: 54: 52-62.

3. Brohi K, Singh J, Heron M, Coats T. Acute traumatic coagulopathy. The Journal of Trauma. 2003: 54(6): 112730 .

4. Misgav M, Martinowitz U. Trauma induced coagulopathy: mechanisms and state of the art treatment. Harefuah. 2011 Feb: 150(2): 99-103.

5. Frith D, Brohi K. The pathophysiology of trauma induced coagulopathy. Curr Opin Crit Care 2012 Dec: 18(6): 631-6.

6. Kutcher ME, Howard BM, Sperry JL, Hubbard AE, Decker AL, Cuschieri J, Minei JP, Moore EE, Brownstein BH, Maier RV, Cohen MJ. Evolving beyond the vicious triad: differential mediation of traumatic coagulopathy by injury, shock and resuscitation. Journal of Trauma Acute Care Surg 2015 Mar: 78(3): 516-23.

7. Hagemo JS, christiaans SC, Stanworth SJ, Brohi K, Johansson PI, Goslings JC, Naess PA, Gaarder C. Detection of acute traumatic coagulopathy and massive transfusion requirements by means of rotational thromboelastometry: an international prospective validation study. Crit Care. 2015 Dec: 19(1): 823 .

8. Cotton BA, Guy JS, Morris JA. Cellular, metabolic, and systemic consequences of aggressive fluid resuscitation strategies. Shock 2006: 26: 115-21.

9. Lier H, Krep H, Schroeder S. The influence of acidosis, hypocalcemia, anemia, and hypothermia on functional hemostasis in trauma. Journal of Trauma 2008: 65: 951-60. 
10. Wyrzykowski AD, Feliciano DV. Trauma damage control. In: Feliciano DV, Mattox KL, Moore EE, editors. Trauma. $6^{\text {th }}$ ed. New York: McGraw-Hill Medical: 2008: 851-70.

11. Cushman JG, Feliciano DV, Renz BM. Iliac vessel injury: Operative physiology related to outcome. Journal of Trauma 1997: 42: 1033-40.

12. Holcomb JB, Jenkins D, Rhee P. Damage control resuscitation: directly addressing the early coagulopathy of trauma. Journal of Trauma 2007: 62: 307-10. 